\title{
Off-axis aberrations of a wide-angle schematic eye model
}

\author{
Isabel Escudero-Sanz \\ Applied Optics, Physics Department, Imperial College, Prince Consort Road, London SW7 2BZ, United Kingdom \\ Rafael Navarro \\ Instituto de Óptica “Daza de Valdés," Consejo Superior de Investigaciones Científicas, Serrano 121, \\ 28006 Madrid, Spain
}

Received November 16, 1998; accepted March 12, 1999; revised manuscript received March 15, 1999

\begin{abstract}
A schematic eye model based on anatomical data, which had been previously designed to reproduce image quality on axis, has been transformed into a wide-angle model by simply adding a spherical image surface that plays the role of the retina. This model captures the main features of the wide-angle optical design of the human eye with minimum complexity: four conic optical surfaces plus a spherical image surface. Seidel aberrations (spherical aberration, coma, astigmatism, field curvature, and distortion), Iongitudinal and transverse chromatic aberrations, and overall monochromatic spot diagrams have been computed for this eye model and for field angles ranging from $0^{\circ}$ to $60^{\circ}$ by both finite and third-order ray tracing. The modulation transfer function for each field angle has been computed as well. In each case our results have been compared with average experimental data found in the literature, showing a reasonably good agreement. The agreement between the model and experimental data is better off axis, mainly at moderate $\left(10^{\circ}-40^{\circ}\right)$ field angles, than on axis. The model has been applied to simulate a variety of experimental methods in which image aberrations are estimated from measurements taken in the object space. Our results suggest that for some types of aberration, these methods may yield biased estimates. (c) 1999 Optical Society of America
\end{abstract}

[S0740-3232(99)00208-2]

OCIS codes: $330.5370,330.4060,080.3630$.

\section{INTRODUCTION}

Schematic eye models that can reproduce optical properties from anatomy are especially useful. They can be used in the design of ophthalmic or visual optics, to simulate experiments, to predict the effect of refractive surgery or implants, or just to understand better the role of the different optical components, among other applications. Furthermore, schematic eyes are necessary even to estimate basic (first-order) optical properties of the eye (i.e., the focal length). The famous Gullstrand eye ${ }^{1}$ (based on an older model by Listing), later updated by Le Grand, ${ }^{2}$ has been highly successful and is still in use nowadays, because it is simple, is based on anatomy, and reproduces the Gaussian properties of an average eye.

From this basic model, there have been two main tendencies. On the one hand, simplified reduced eyes have been derived. Although Le Grand ${ }^{2}$ pointed out that it is necessary to avoid the use of the reduced eye because such a scheme is too crude, reduced eyes can be specifically designed to reproduce some ocular aberrations ${ }^{3,4}$ or even as wide-angle models to reproduce oblique astigmatism. $^{5}$

On the other hand, other authors have attempted to follow anatomy more accurately, ${ }^{6-8}$ incorporating a gradient-index (GRIN) crystalline lens, which is sometimes approximated by a shell structure. ${ }^{9}$ Nevertheless, the exact distribution of the refractive index of the human lens is not well known yet, and these models tend to have several adjustable parameters. Less attention has been paid to including other anatomical features in eye models, such as decentering of lenses and iris or other nonaxially symmetric features that potentially have a strong effect on optical performance. In fact, rotationally symmetric eyes are incomplete in the sense that they predict only spherical and chromatic aberration in the fovea, whereas real eyes display astigmatism, coma, and irregular aberrations as well. ${ }^{10-13}$

These opposite tendencies demonstrate that there is no general agreement in modeling the eye. The joint anatomical-optical type of modeling is still open for research and discussion. Our approach is to try to harmonize these opposite tendencies with a schematic eye model that can offer a trade-off between accuracy and economy (simplicity). I deally, an eye model should reproduce accurately both anatomy and optical properties (first order, aberrations, and image quality). However, such a model could eventually become highly complicated yet not be useful because of low accuracy. Maybe one would find that there is a lack of reliable experimental data on important parameters necessary to build the model and end up with a model of not much use in practice because of the high intersubject variability.

The Gullstrand-Le Grand eye accomplishes the abovementioned trade-off, except that it exhibits substantially higher aberrations than normal emmetropic eyes. To better predict optical performance, spherical 
aberration, ${ }^{4,8,14}$ and oblique astigmatism ${ }^{15-17}$ or to build wide-angle eye models, ${ }^{18}$ it is necessary to include aspheric surfaces. However, it is not possible to predict the optical performance of real human eyes on axis with a rotationally symmetric model. Nevertheless, the great majority of schematic eyes have axial symmetry, thus allowing prediction of only chromatic and spherical aberrations on axis. Many authors have attempted to measure the spherical aberration of the eye, often finding that the aberration changes with the orientation (or the meridian). This lack of rotational symmetry suggests the presence of other aberrations, since spherical aberration is rotationally symmetric by definition. However, one should bear in mind that a simple pupil decentering can convert part of the spherical aberration and defocus into coma and astigmatism and that any nonaxially symmetric feature or local defect will yield an overall aberration that may be far from having even symmetry. Nevertheless, if we consider a large population of different eyes and assume that departures from axial symmetry are randomly but uniformly distributed, the average of their aberration pattern will tend to be symmetric. In this sense a schematic eye model may reproduce the expected amount of aberration but not the highly irregular patterns found in individual eyes.

One can even argue that a wide-angle model should be able to reproduce the main features of off-axis performance but not try to fit exactly each aberration at every retinal location. The latter would be a difficult task to undertake, and besides there are not enough experimental data to even attempt it. In fact, only a few authors have attempted to reproduce off-axis aberrations with wide-angle model eyes (except for oblique astigmatism ${ }^{16,17}$ or chromatic aberration ${ }^{19}$ ). One important reason was simply that no data on some aberrations, such as peripheral coma, were available.

Thus the first goal of this work is to build a wide-angle model, departing from a simple schematic eye, that reproduces on-axis optical performance reasonably ${ }^{14}$ in order to reproduce off-axis performance by adding a minimum of changes. We believe that this is one of the first attempts to reproduce aberrations and overall performance across field angle, and we think that it is worth trying. A secondary goal, which is a potentially interesting application of this wide-angle model, is to compare aberrations in the object and image spaces. Thibos et al. ${ }^{4}$ have already pointed out the difference between the object and image spaces in the particular case of spherical aberration. The knowledge of object aberrations is of interest for applications such as fundus imaging, where the retina is viewed through the optical system of the eye, but it is more important to know image (retinal) aberrations, since they limit visual perception. However, aberrations are, in most cases, measured in the object space instead of in the image space, because the retinal image is not accessible easily, and only very few methods, among those proposed in the literature, measure aberrations in the image space subjectively ${ }^{10}$ and objectively. ${ }^{12}$ In this sense the model eye permits us to compute aberrations in either the object or the image space and may help to estimate the potential biases of experimental measurements of ocular aberrations by subjective and objective techniques.

\section{WIDE-ANGLE SCHEMATIC EYE}

The eye model proposed and studied here is an extension of that by Navarro et al., ${ }^{14}$ which is similar to Kooijman's wide-angle model. ${ }^{18}$ That model was built by incorporating published conic constant anatomical values into the Gullstrand-Le Grand spherical surfaces ${ }^{2}$ and updating the value of the anterior radius and the refractive index of the cornea with the use of more recent anatomical data. The same refractive indices as those in Ref. 2 were used for the other ocular media and for the standard D line (589.3-nm wavelength). Refractive indices for other wavel engths were estimated, departing from experimental data of chromatic dispersions and adjusting experimental values of the longitudinal chromatic aberration (LCA) (see Ref. 14 for details).

The optical quality of that schematic eye (spherical and chromatic aberrations, as well as polychromatic pointspread functions and modulation-transfer functions (MTF's) have already been studied in Ref. 14 but only on axis. One important feature of that model, which has been kept here, is that all parameters that define its geometry are anatomical, and there was no need to fit or change original values to reproduce average data on spherical aberration. Refractive indices, especially for $\lambda=589.3 \mathrm{~nm}$, were also anatomical, except for the GRIN structure of the lens. In that case the equivalent constant index used by Gullstrand and Le Grand to reproduce the refractive power of the lens was adopted here.

To extend this on-axis schematic eye to a wide-angle model, we have made a small modification: the addition of a curved image surface (the retina) having the simplest shape, i.e., spherical, with a $12-\mathrm{mm}$ radius of curvature. ${ }^{2}$ This image surface is intersected by the optical axis at the paraxial focus for $543 \mathrm{~nm}$, which has been the reference wavel ength in this work. (This choice is arbitrary, but it is appropriate to compare the model predictions with recent experimental data that are being obtained in differ-

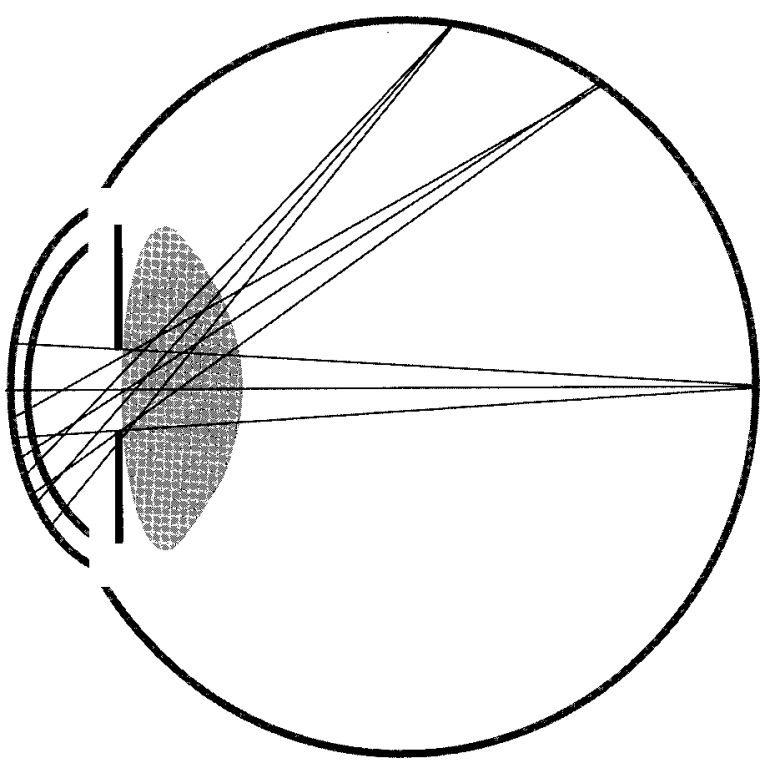

Fig. 1. Plot of the schematic model of the human eye of Table 1 with a 3-mm entrance pupil diameter. Rays for three field objects at infinity are traced: minimum $\left(0^{\circ}\right)$, intermediate $\left(40^{\circ}\right)$, and maximum $\left(60^{\circ}\right)$ fields considered in this study. 
Table 1. Geometry of the Schematic Wide-Angle Eye Model

\begin{tabular}{clcccc}
\hline Surface & Type & Conic Constant & Radius $(\mathrm{mm})$ & Thickness $(\mathrm{mm})$ & Optical Medium \\
\hline 1 & Conic & -0.26 & 7.72 & 0.55 & Cornea \\
2 & Spherical & 0 & 6.50 & 3.05 & Aqueous \\
Stop & Plane & 0 & Infinity & 0 & Aqueous \\
4 & Conic & -3.1316 & 10.20 & 4.00 & Lens \\
5 & Conic & -1.0 & -6.00 & 16.3203 & Vitreous \\
Image & Spherical & & -12.00 & & \\
\hline
\end{tabular}

Table 2. Refractive Indices for the Wavelengths Used in Computations

\begin{tabular}{lcccc}
\hline Medium & $458 \mathrm{~nm}$ & $543 \mathrm{~nm}$ & $589.3 \mathrm{~nm}$ & $632.8 \mathrm{~nm}$ \\
\hline Cornea & 1.3828 & 1.3777 & 1.376 & 1.3747 \\
Aqueous & 1.3445 & 1.3391 & 1.3374 & 1.336 \\
Lens & 1.4292 & 1.4222 & 1.42 & 1.4183 \\
Vitreous & 1.3428 & 1.3377 & 1.336 & 1.3347 \\
\hline
\end{tabular}

ent laboratories with green $\mathrm{He}-\mathrm{Ne}$ lasers. In addition, this wavelength is close to the maximum of the spectral luminous efficiency function for the standard observer $\left(V_{\lambda}\right): \lambda_{\max } \approx 555 \mathrm{~nm}$.) The schematic eye is depicted in Fig. 1, and Tables 1 and 2 show the geometrical parameters and the refractive indices, respectively. For $\lambda$ $=543 \mathrm{~nm}$, the focal length of this schematic eye is 22.03 $\mathrm{nm}$ in the image space (the effective focal length in air is $16.47 \mathrm{~mm}$ ), and the refractive power is 60.7 diopters (D). The entrance pupil position is $3.04 \mathrm{~mm}$ from the first surface, and the exit pupil position is $-3.92 \mathrm{~mm}$ from the back surface of the eye lens.

All ray tracing, wave-front analysis, and MTF computations have been carried out with Zemax optical design software. Third-order calculations are based on the Seidel wave-front coefficients through paraxial ray tracing of marginal and chief rays. ${ }^{20}$

In what follows, aberrations have been estimated in three ways (in most cases): (1) by finite ray tracing, (2) by computation of third-order Seidel coefficients, and (3) by simulation of experiments taken from the literature by finite ray tracing (usually by computing aberrations in the object space). In several cases our schematic eye predicts substantial offsets between aberrations in the object space (simulating experimental data) and in the image space computed by applying standard definitions.

\section{ABERRATIONS: MODEL PREDICTIONS AND EXPERIMENTAL DATA}

In this section the aberrations of the schematic eye introduced in Section 2 are computed and compared with experimental data published in the literature. As we said above, three different calculations of the aberrations of the model have been carried out that include aberrations computed by modeling experiments described in the literature. In this way we can (1) test how well the model reproduces the optical quality of real eyes on and off axis and (2) predict biases between experimentally measured aberrations with different techniques and theoretical image-domain (retinal) values. Unless stated otherwise, standard calculations have been carried out for light of 543-nm wavelengths. Results for longitudinal aberrations (defocus, spherical aberration, astigmatism, etc.) are given in terms of differences in refractive power in diopters, and transverse aberrations (coma, transverse color, spot diagrams, etc.) are given as angular displacements in arc minutes.

\section{A. Spherical Aberration}

Spherical aberration has been widely studied in the literature and still is one of the most controversial subjects regarding aberrations of the eye. Not only the absolute value of this aberration but also its relative contribution to the overall ocular optical performance is a source of disagreement. On the one hand, specific methods for the direct measurement of the longitudinal spherical aberration $(\mathrm{LSA})^{21}$ tend to yield large amounts of aberration (typically 2-3 D for 8-mm pupil diameter); thus these studies attribute a major role to this aberration in the overall performance of the system on axis. On the other hand, relatively small values of spherical aberration are obtained with experimental methods designed to measure the overall wave-front aberration, ${ }^{10-13}$ thus leading to the opposite conclusion. Moreover, in the latter type of study, Zernike coefficients of astigmatism and coma are more important than the corresponding spherical aberration coefficients in individual eyes. We are aware of this controversy but here have adopted the experimental values given by the first type of method because we believe that they better represent average data (over subjects but typically also over orientations), whereas overall wavefront data better describe individual eyes. Furthermore, Charman and Walsh ${ }^{22}$ averaged wave aberration experimental data over all meridians and estimated the resulting geometrical "spherical" aberration. They compared their results with those of earlier authors for the spherical aberration, concluding that the results for both kinds of measurements agree reasonably well.

The LSA has been computed for a maximum entrance pupil of $8 \mathrm{~mm}$, and the results are given in Fig. 2. It includes third-order (Seidel, dashed curve) and finite raytracing computations of the LSA for the schematic eye model (solid curve) and computer simulations of the experiments by Koomen et al., ${ }^{21}$ as well as the experimental values obtained by these authors (triangles), data from several authors compiled by van Meeteren ${ }^{23}$ (solid squares), and values obtained by Thibos et al. ${ }^{4}$ (open circles). The units are diopters, that is, the aberration measured as the difference between the reciprocals of optical distances $(n / l)$. (For instance, the aberration in our calculations is the product of the refractive index $n^{\prime}$ with 
the difference between the reciprocal of the paraxial focal length and the reciprocal of the distance between the image principal plane and the focus of the rays through a given pupil radius.) We can see that the agreement of our computations with the data of van Meeteren and Thibos et al. is almost perfect. However, it is important to remember the different natures and origins of these sets of data: van Meeteren's data are the result of a curve fitting to the experimental data available in the literature at that time; Thibos et al. adjusted the shape of a singlesurface reduced eye to reproduce experimental values of the LSA; in contrast, our schematic eye reproduces the same LSA by using anatomical data without any fitting.

To simulate the experiment by Koomen et al., ${ }^{21}$ we have introduced annular apertures placed $5 \mathrm{~mm}$ in front of the cornea into our model eye. In their experiment, which we have simulated here, these apertures had different radii so that the pupil could be sampled in the radial variable but rays could be integrated in the angular variable. For each pupil radius (aperture), the distance from the point object to the eye was changed until the image was on focus at the retina. The conjugate lens equation $\left(-n / l+n^{\prime} / I^{\prime}=n^{\prime} / f^{\prime}\right.$, where $n$ and $n^{\prime}$ are the refractive indices of air and aqueous humor, respectively; $f^{\prime}$ is the focal length; and I and I' are the distances from the object and the image to the corresponding object and image principal planes, respectively), is then used to find the value of the spherical aberration for that radius. Writing this equation, both for the data obtained for a given radius $(r)$ and for the reference paraxial case $(p)$, subtracting both expressions and using the fact that the image distance is fixed $\left(\mathrm{I}^{\prime}=\right.$ constant $)$ and that it does not change with radius, we find that the LCA in diopters for a given radius is

$$
\operatorname{LCA}(r)=\left(n^{\prime} / f_{r}^{\prime}\right)-\left(n^{\prime} / f_{p}^{\prime}\right)=\left(n / l_{p}\right)-\left(n / l_{r}\right),
$$

where $I_{p}$ and $I_{r}$ are the object distances to focus the image for the paraxial circle $(r=0)$ and for the ring of radius $r$, respectively. It can be seen in Fig. 2 that experimental values by Koomen et al. (triangles) are slightly above the results of our computer simulation (solid circles), but the

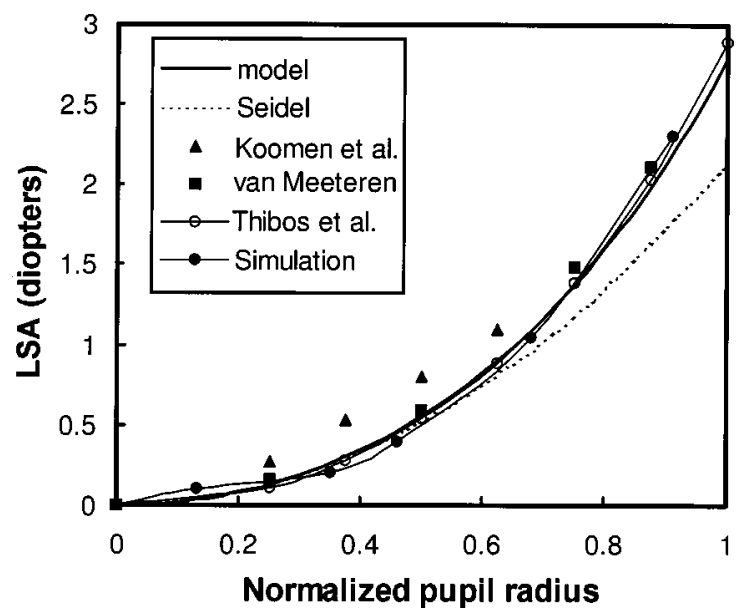

Fig. 2. Longitudinal spherical aberration (LSA) of the schematic eye with an 8-mm-diameter entrance pupil, compared with experimental data taken from the literature, in diopters (see the symbol key and the text for details).

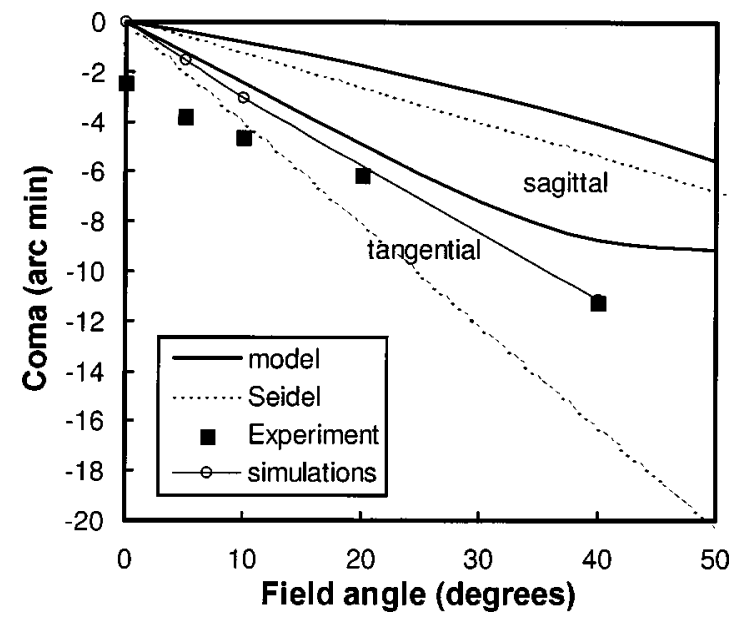

Fig. 3. Tangential and sagittal angular coma, in arcminutes, of the schematic eye, compared with experimental tangential coma.

overall agreement is reasonable and is much better if we consider average data. ${ }^{4,23}$ Therefore, if one considers spherical aberration as the angular average of ocular aberrations, as Koomen et al. and others did, then real eyes seem quite well represented by the schematic model. Equally, the results of this simulation and the actual spherical aberration of the model (solid curve) are very much alike, which indicates the validity of this kind of experiment. We want to recall that in the schematic eye model ${ }^{14}$ the conic constants (aspheric surfaces) control the LSA, which is much lower than in the Gullstrand eye (spherical surfaces). This aberration control includes higher-order LSA, so that the third-order Seidel (e.g., see Ref. 20) term yields a good approximation of the total LSA for pupil diameters up to $6 \mathrm{~mm}$.

\section{B. Coma}

Until recently there was a lack of experimental data of coma except at the fovea. As we said above, the current version of our schematic model is rotationally symmetric and thus does not display coma on axis, but here we are interested in studying the schematic model as a wideangle eye and hence its off-axis aberrations. As far as we know, there is only one recent, systematic study of the offaxis overall monochromatic aberrations in the human eye. $^{24}$ In that study, aberrations are measured at the image plane by a laser ray-tracing technique, thus being directly comparable with the finite ray-tracing computations carried out here. Nevertheless, other methods, either objective ${ }^{11}$ or subjective, ${ }^{25}$ measure aberrations in the object space. We have simulated the latter, subjective methods, which are based on measurements of the change from the direction of rays (coming from a point object at infinity) in the object space to the direction at which the rays intersect the principal ray at the image surface (the retina).

The results of computations of angular coma, for marginal rays in an 8-mm entrance pupil, are presented in Fig. 3. The third-order tangential and sagittal coma, derived from Seidel coefficients ${ }^{20}$ (dotted curves), are compared with the more exact values (solid curves), obtained as the difference between the angle of finite marginal rays in the image space and the angle of an ideal ray, which 
would form a nonaberrated image. In the case of tangential coma, the aberration is the mean of the values of diagonally opposite marginal rays. The figure also includes a simulation of what would be expected in the subjective experiment (circles), which predicts a small but significant bias, tending to overestimate tangential coma. Interestingly, we can appreciate in the figure how thirdorder coma is larger than the actual value (especially tangential coma). This suggests that the optical design of the eye is helping to maintain aberrations within moderate levels: it seems clear that higher-order terms are playing a compensating role, helping to control this aberration. Average experimental data of third-order tangential coma, estimated from Zernike coefficients, ${ }^{24}$ are represented as squares. We can see that experimental data differ from those of the model for zero or small field angles, simply because the symmetric model cannot predict any coma on-axis. However, the two sets of data tend to merge, which results in a much better agreement from $20^{\circ}$ to $40^{\circ}$. Thus the model seems to be capable of capturing the overall wide-angle behavior, that is, the field aberration, but it cannot reproduce on-axis coma unless it incorporates some asymmetric features.

\section{Astigmatism}

Astigmatism has been computed as the difference between the reciprocals of sagittal and tangential focal lengths multiplied by the refractive index of the vitreous. These are effective lengths given as the distance from the image (second) principal plane to the focus (sagittal or tangential) measured along the principal ray. Thirdorder Seidel coefficients were also computed. Figure 4 compares our results with averaged experimental data (solid symbols). These averages were estimated by Lotmar and Lotmar ${ }^{16}$ from original data by Rempt et al. ${ }^{26}$ In that experiment the aberration is found by moving the object back and forth until the tangential and sagittal images are formed at the retina. The conjugate formula is then applied in a way similar to that for spherical aberration. In this case the distances to the sagittal and tan-

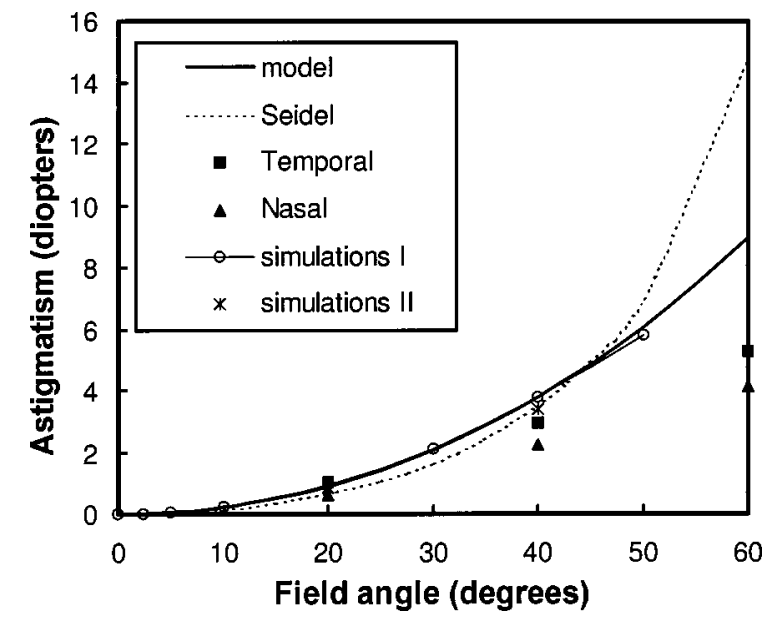

Fig. 4. Oblique astigmatism of the eye model compared with experimental data. Results of two computer simulations of experimental measurements, moving the object distance (I) and using trial cylinder lenses (II), are included. gential images are identical (since both images are at the retina). Thus the astigmatism is given by

$$
\left(n^{\prime} / f_{t}\right)-\left(n^{\prime} / f_{s}^{\prime}\right)=\left(n / I_{s}\right)-\left(n / I_{t}\right),
$$

where $f_{s}^{\prime}$ and $f_{t}^{\prime}$ are the sagittal and tangential effective focal lengths and $I_{s}$ and $I_{t}$ are the distances to the object that produce the sagittal and tangential images at the retina. It is important to note here that the conjugate equation does not need to be valid outside the paraxial region, as will be discussed further in Subsection 3.D in the case of field curvature. We have simulated this experiment (simulation I), and the results are displayed as circles.

A second simulation (II) considers that optometrists use cylindrical lenses to measure and compensate for astigmatism. This method has been simulated for field angles of $20^{\circ}$ and $40^{\circ}$ (asterisks). A simulated cylindrical lens was placed $5 \mathrm{~mm}$ in front of the cornea and oriented and centered in such a way that the principal ray is coincident with the normal to the lens at each field angle. Simulations predict slightly lower values of astigmatism, mainly for the latter method. The agreement with experimental data is reasonable up to $40^{\circ}$ of visual field, whereas for higher eccentricities our model predicts higher values. In addition, the third-order calculations are a good enough approximation, except for very large eccentricities, where higher-order terms again seem to partially compensate for third-order astigmatism. It would appear that both experimental methods are adequate to measure astigmatism, since they do not produce substantial biases. In conclusion, there is a good agreement between experimental values and theoretical ones computed from our model for small and moderate field angles, but there is an increasing mismatch for very large field angles.

\section{Field Curvature}

When an optical system has field curvature, the actual image surface departs from the paraxial image surface as though the images of off-axis objects were increasingly out of focus. Therefore this aberration can be represented by the amount of defocus of the images or, in other words, by the departure of the actual image surface, given by the geometrical locus of the disks of least confusion, from the reference surface. To estimate the field curvature in the schematic model eye, we have set the reference surface to be the (spherical) retina in all cases. This also applies to third-order calculations, for which the curvature radius of the image surface has been found by using the Seidel coefficients for astigmatism and Petzval curvature. The results, given in Fig. 5, are compared with experimental data by Rynders et al. ${ }^{27}$ (squares). The results obtained by simulating that experiment (circles) show an excellent agreement with the average experimental data. The experiment is conceptually similar to the one described in Subsection 3.C for astigmatism: The object moves back and forth until its image, the disk of least confusion, is focused at the retina. However, the simulation also predicts a moderate offset (increasing with field angle) between measured (objectspace) curvature and retinal (image-space) curvature. Again, third-order Seidel curvature provides a good ap- 


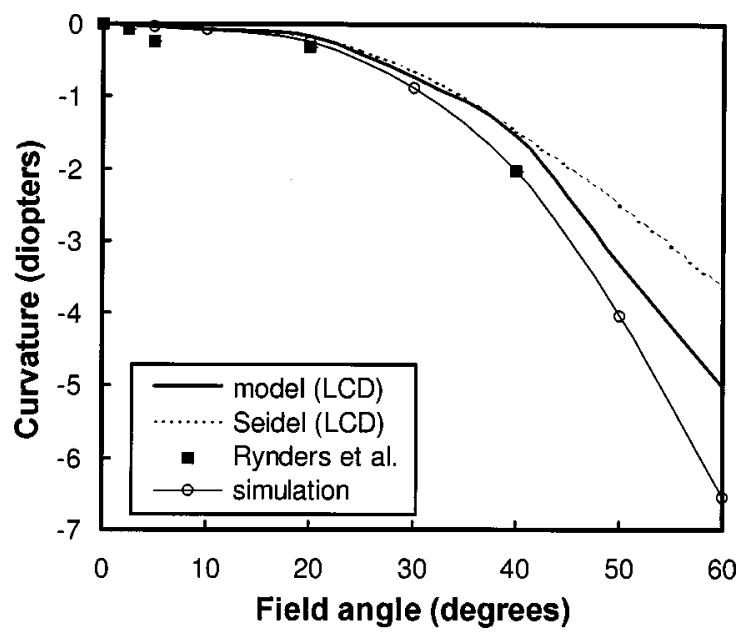

Fig. 5. Field curvature of the eye model, estimated as the offset, in diopters, between the least confusion disk (LCD) and the retina (solid curve); third-order LCD (dotted curve); experimental data (solid squares); and computer simulation of the experiment (open circles).

proximation to the total value for field angles up to $40^{\circ}$. In this case the curvature of the retinal surface, instead of higher-order terms, is the major force controlling this aberration.

Figure 6 compares the curvature of the retinal surface versus the different image curvatures mentioned above. These are the Petzval surface [a 17-mm-radius sphere (asterisks)], the third-order image surface [a 13-mmradius sphere (circles)], the image surface obtained by finite ray tracing (solid curve), and the retina [a $12-\mathrm{mm}$ radius sphere (dotted curve)]. The origin of coordinates is the paraxial focus at $\lambda=543 \mathrm{~nm}$. Under this representation the good overall match between both retinal and image surfaces appears much clearer. Although our spherical retinal surface is a rather crude approximation to the actual shape of the retina in individual eyes, it captures the essential fact that retinal curvature seems well adapted to compensate for the curvature of the image surface.

Analysis of Figs. 5 and 6 leads to the conclusion that this schematic model is a good representation of the eye, as the good agreement between experimental and simulated data shows. It is worth noting that field curvature often is the most important aberration in a wide-angle optical system. If there were not a good match between the optical image and the retinal surface, defocus could reach huge values in the periphery (as suggested by Fig. 6). Thus a wide-angle model that provides a good agreement with experimental field curvatures, such as this one, as well as a reasonable fit to the order of magnitude of other aberrations, will be able to make good predictions of the overall optical quality of an average eye. Figure 5 suggests that the experimental method followed by Rynders et al. ${ }^{27}$ tends to overestimate field curvature at large eccentricities, probably because it uses the conjugate equation far off the paraxial region. Figures 5 and 6 together reveal an interesting feature of the optical design of the eye: The astigmatism contributes to the correction of field curvature in the sense of bending the image surface closer to the retina rather than in the sense of flattening the field (as happens in a Petzval lens, for instance). This is shown clearly in Fig. 6, where the departure of the Petzval surface from the retina is larger than that of the surface containing the disks of least confusion. The experimental values of astigmatism and field curvatures (Figs. 4 and 5) also support this idea, suggesting that this joint compensation (astigmatism and retinal curvature) could actually be taking place in nature.

\section{E. Distortion}

Although it is hard to estimate distortion in the human eye experimentally, we include predictions of our eye model here (Fig. 7) to complete our analysis. Again, it is clear that higher-order terms tend to compensate for third-order distortion in the eye model, mainly for higher eccentricities, where the overall distortion shows moderate values.

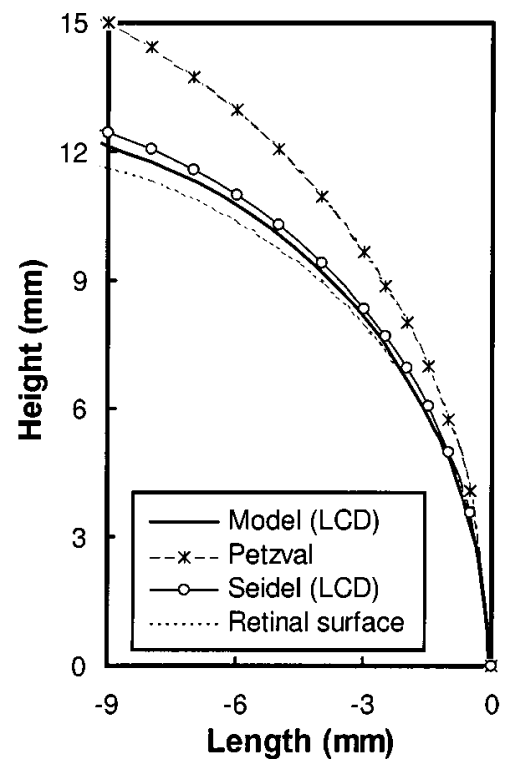

Fig. 6. Shape and relative location of the different image surfaces of the eye: Model surface (solid curve), computed as the locus of the disks of least confusion for finite ray tracing with the use of a 3-mm pupil, is compared with the Petzval surface (asterisks), with the disks of least confusion in third-order approximation, and finally with the retinal surface that is approximated by a sphere in the eye model.

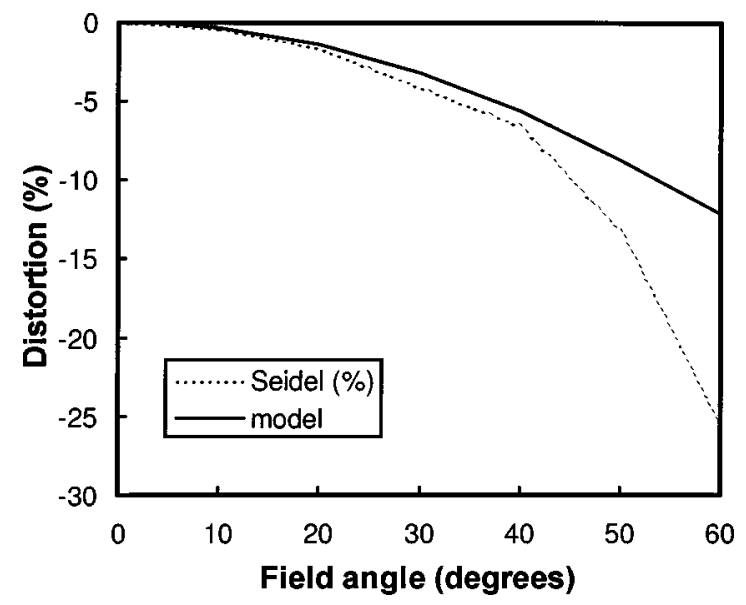

Fig. 7. Angular distortion of the eye model. (We have not found experimental data available.) 


\section{F. Longitudinal Chromatic Aberration}

Longitudinal color, chromatic difference of focus, and LCA of this schematic eye model have been reported previously, ${ }^{14}$ showing a close match with experimental data. Although the LCA is not expected to change much with field angle, this could be somewhat different for large field angles because of potential changes in the effective refractive power. In a recent work by Rynders et $a l .,{ }^{27}$ the LCA has been measured as a function of visual field, yielding a slightly higher LCA for large visual angles. Here we report a simulation of that experiment with the same wavelengths-632.8, 543, and $458 \mathrm{~nm}-$ that were used in the experiment. As we said in Subsection 3.D, that experiment makes use of the conjugate equation to estimate the LCA, even though the experimental conditions did not guarantee the validity of the Gaussian approximation. The procedure consisted of changing the object distance to obtain the best image quality (focus) at the retina. When we write the conjugate equation for two colors (red and blue, for instance) and simplify the term corresponding to the image distances, the image plane LCA between these wavelengths is given by

$$
\left(n_{r}^{\prime} / f_{r}^{\prime}\right)-\left(n_{b}^{\prime} / f_{b}^{\prime}\right)=\left(n_{b} / I_{b}\right)-\left(n_{r} / I_{r}\right) .
$$

This expression was also used here to simulate the experiment. The results are presented in Fig. 8. These include the variation of chromatic difference of effective power (which corresponds to the effective focal length defined in Subsection 3.C), that is, the LCA of the eye model as a function of field angle (solid curve); experimental data (squares; error bars indicate intersubject variability); the results of simulating this experiment with the model eye (curve with circles); and, finally, the results of the same simulation, but now including a correction term (dotted curve with asterisks). This correction term takes into account that not only the refractive power but also other Gaussian parameters, such as the positions of the principal planes of the eye model, varies with color. If we take into account that the position of the image principal plane does not change with wavelength, we arrive at the correct expression that links the LCA with measurements carried out in the object space:

$$
\begin{aligned}
\left(n_{r}^{\prime} / f_{r}^{\prime}\right)-\left(n_{b}^{\prime} / f_{b}^{\prime}\right)= & \left(n_{b} / I_{b}\right)-\left(n_{r} / I_{r}\right) \\
& +\left(n_{r}^{\prime} / I_{r}^{\prime}\right)-\left(n_{b}^{\prime} / l_{b}^{\prime}\right) .
\end{aligned}
$$

This is a typical example in which an eye model can be of valuable help. The correcting term $\left(n_{r}^{\prime} / l_{r}^{\prime}\right)$ - $\left(n_{b}^{\prime} / l_{b}^{\prime}\right)$, could not be determined experimentally, but the eye model can provide an estimation, thus permitting further refinements of the LCA measurements. In fact, we can see in Fig. 8 that the experimental values (squares) and the results of the simulation (without correcting term) (circles) are similar (simulation results are within the range covered by experimental error bars, except for an anomalous point at $5^{\circ}$ ), but both differ from the image-space LCA estimated from the model. The reason for this discrepancy is that the experimental method measures the aberration in the object space, that is, the amount $\left(n_{b} / I_{b}\right)-\left(n_{r} / I_{r}\right)$. If we want to estimate the aberration in the image space from these experimen- tal data, it is necessary to know the correcting term $\left(n_{r}^{\prime} / I_{r}^{\prime}\right)-\left(n_{b}^{\prime} / l_{b}^{\prime}\right)$, which accounts for changes in the position of the image principal plane with wavelength. If we can determine it (by experiment or simulation), then Eq. (4) permits a better approximation to the LCA. We can see in Fig. 8 that the agreement between the model, image-space LCA (solid curve) and the data of the corrected simulation, that is, the data obtained by computing the LCA in the object space and then applying Eq. (4) (asterisks), is much closer. However, the results of the uncorrected simulation (open circles) are consistent with the experimental data that were not corrected. In this case there is a substantial bias between the aberrations in the object and image spaces.

\section{G. Transverse Color}

The computation of the transverse chromatic aberration (TCA) with finite ray tracing involves finding the change in direction of the principal ray in the image space for different wavelengths. The experimental data reported in the literature have been obtained by applying the same idea, but as in many other cases, the change in direction can be measured only in the object space. Despite the sparse literature available measuring the TCA across the visual field, there are large discrepancies in the experimental data reported by different authors. Thibos et al. ${ }^{19}$ discard the Ogboso and Bedell data, ${ }^{28}$ citing their large experimental errors, but, nevertheless, we include both sets of data here. These experimental data, along with the results of the eye model and simulations, are shown in Fig. 9. This figure contains six sets of data: transverse color of the schematic eye model, obtained by finite ray tracing at $572 \mathrm{~nm}$ and $435 \mathrm{~nm}$, the wavelengths used by Ogboso and Bedell ${ }^{28}$ (thick solid curves); data obtained in the simulation of that experiment with the above wavelengths (curve with open circles); the original experimental data ${ }^{28}$ obtained, along both the temporal (solid circles) and nasal (squares) meridians; data from Thibos et al. ${ }^{19}$ for wavelengths $433 \mathrm{~nm}$ and $622 \mathrm{~nm}$, corresponding to an experimental simulation in which the off-axis TCA was inferred from measurements of the

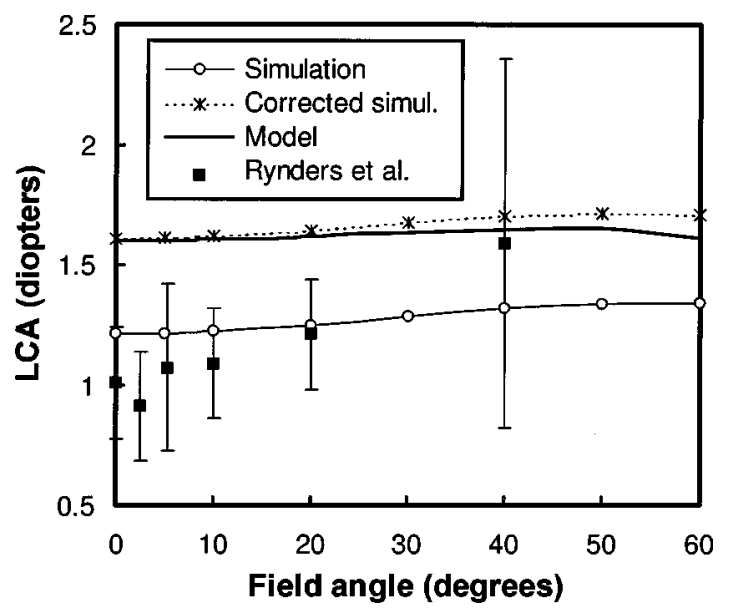

Fig. 8. Variation of longitudinal color (LCA) with field angle for the schematic eye model (solid curve), along with experimental data (solid squares) and simulations of that experiment, both direct simulation (open circles) and corrected from estimated bias (asterisks). 


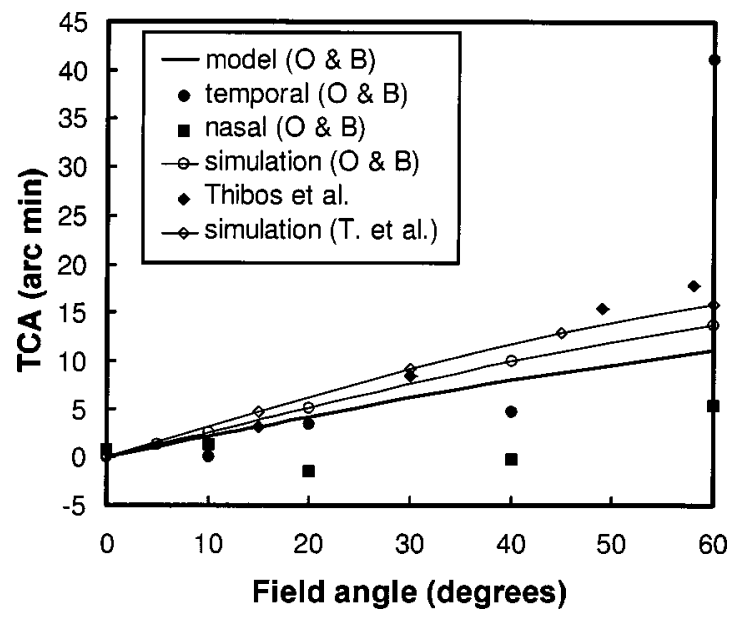

Fig. 9. Transverse color TCA for the model, experimental data, and computer simulations.

foveal TCA as a function of the displacement of an artificial pupil (solid diamonds); and our simulation of the TCA for the wavelengths used in the latter experiment (open diamonds). From this figure we can extract several conclusions. Simulated experiments (that is, object-space TCA) predict a moderate overestimation of the TCA in the image space given by the model. The offset between the object and image values is approximately proportional to the value of the TCA. There is a close agreement between experimental and simulated data for the Thibos et al. study, which suggests that (1) experimental data could be biased in a similar manner to that of simulation and (2) if that is true, the schematic eye yields a good prediction of the off-axis TCA. Furthermore, at large field angles, the agreement found here is even better than that obtained with the model developed by the authors ${ }^{19}$ to fit the data of the experiment. However, the agreement is worse with the results of the experiment by Ogboso and Bedell.

\section{OVERALL PERFORMANCE}

Spot diagrams, shown in Figs. 10 and 11, have been computed to provide a more general view of the ability of the schematic eye model to reproduce the overall optical performance of the eye as a wide-angle optical system. Again, in the computations, we have tried to follow experimental conditions for the data available in the literature. As far as we know, there is only one recent study ${ }^{24}$ reporting experimental spot diagrams across the visual field, and thus we have reproduced those ray-tracing conditions in Fig. 10: 1-mm sampling interval in a square grid, 6-mm pupil diameter, and 543-nm wavelength. The results for $0^{\circ}, 5^{\circ}, 10^{\circ}, 20^{\circ}, 40^{\circ}$, and $60^{\circ}$ of field are shown in Fig. 10. Each box represents $300 \times 300 \mu \mathrm{m}$ at the retina (300 $\mu \mathrm{m} \approx 1^{\circ}$ of field). If we compare these diagrams with experimental data (results for four subjects and for the same visual angles, except for $60^{\circ}$, can be found in Fig. 2 of Ref. 24), there is a notable agreement in the overall features in spite of large intersubject differences in experimental data. Namely, the overall size of the spot diagrams is similar, and the change of optical quality with field angle is slow and gradual, as one would like to have in a wide-angle lens. In addition, the patterns provided by the model strongly resemble some individual patterns found experimentally. Spherical aberration (on-axis), coma, and astigmatism are apparent in Fig. 10. Although field curvature is not really seen, images with less astigmatism could be obtained at large field angles for slightly larger radii of the image surface, whereas no significant change is observed at any field angle if a defocus is introduced. There is some field curvature present, but astigmatism is the dominant effect at large field angles while coma is the dominant effect at moderate angles.

Figure 11 shows spot diagrams for smaller (3-mm) and larger (9-mm) pupil diameters for $0^{\circ}$ and $40^{\circ}$ field angles. For smaller pupils the spot diagram is very small on-axis, since the model eye is nearly diffraction limited, but the image quality declines with eccentricity because of astigmatism and coma. For large apertures (9-mm pupil, not shown here), spherical aberration is the limiting aberration, making the spatial resolution almost uniform over all the field.

Figures 12(a) and 12(b) display the MTF of the schematic eye for field angles $0^{\circ}$ and $20^{\circ}$, respectively. In this case the calculations were carried out at $\lambda=632.8 \mathrm{~nm}$ and for a 4-mm-diameter entrance pupil in order to com-

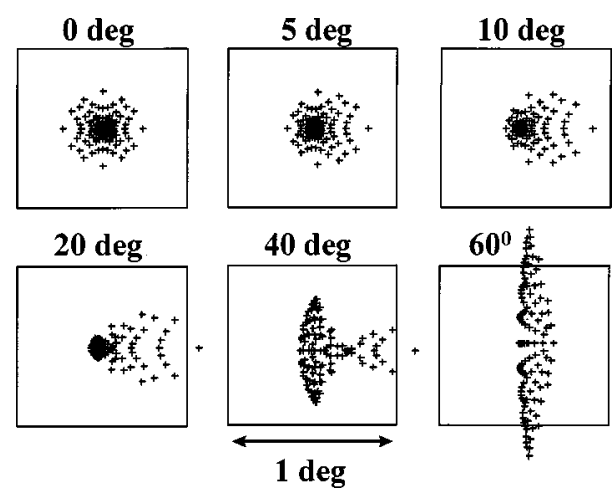

Fig. 10. Spot diagrams for the schematic eye, obtained by finite ray tracing, for a 6-mm entrance pupil diameter and for six field angles: $0^{\circ}, 5^{\circ}, 10^{\circ}, 20^{\circ}, 40^{\circ}$, and $60^{\circ}$.

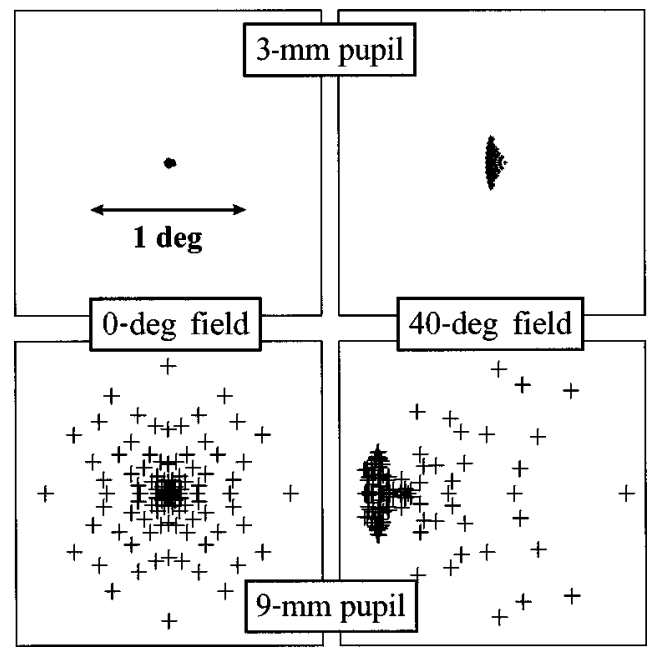

Fig. 11. Spot diagrams for 3-mm and 9-mm pupil diameters and for $0^{\circ}$ and $40^{\circ}$ field angles. 


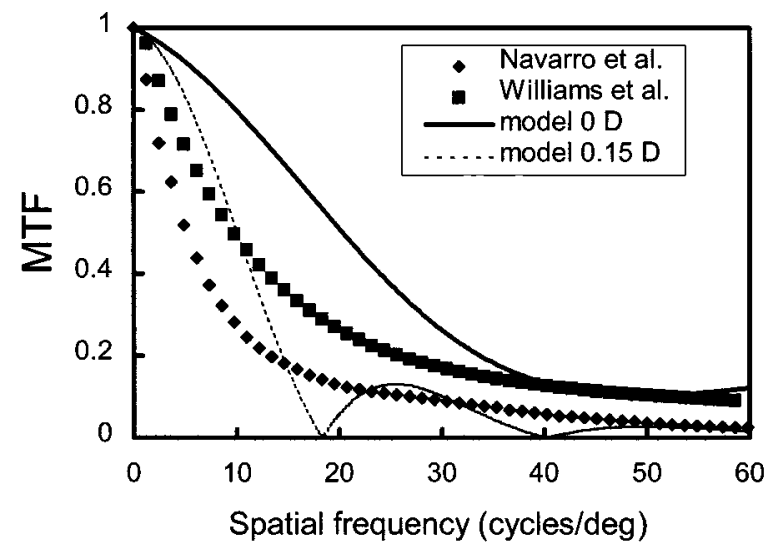

(a)

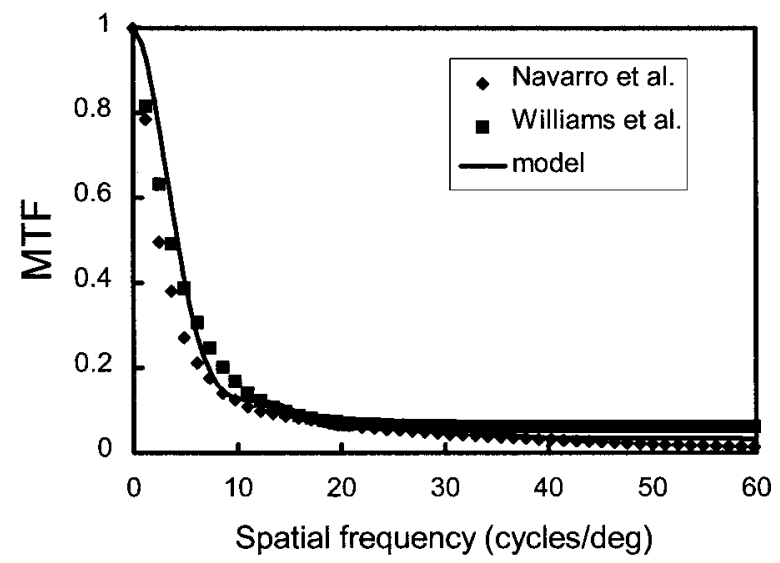

(b)

Fig. 12. Radial profiles (orientation average) of the MTF of the eye model compared with experimental data available in the literature. (a) On-axis. Here the MTF has been computed for 0 and $0.15 \mathrm{D}$ of defocus. (b) $20^{\circ}$ off-axis.

pare the results with experimental values. ${ }^{29}$ In both plots the MTF of the model, averaged across orientations, is compared with two sets of experimental data. ${ }^{29,30}$ These two experiments differ in wavelength, $632.8 \mathrm{~nm}$ versus $543 \mathrm{~nm}$; pupil diameter, $4 \mathrm{~mm}$ versus $3 \mathrm{~mm}$; and in experimental conditions, natural viewing conditions versus best image plane with paralyzed accommodation. Therefore they yield somewhat different results, mainly on-axis. We can see that while the agreement between model and experimental data is quite good off axis $\left(20^{\circ}\right)$, it is worse on axis. In the last case, the MTF for $0.15 \mathrm{D}$ of defocus has been included, following van Meeteren's suggestion that the effect of irregular aberrations-coma, etc.-on the MTF would be equivalent to that of $0.15 \mathrm{D}$ of defocus. We can see that the MTF data of Williams et al. ${ }^{30}$ lie between both theoretical curves (except at very Iow frequencies), tending to fit the theoretical MTF for zero defocus at higher frequencies, whereas the data of Navarro et al. ${ }^{29}$ tend to fit the theoretical MTF for $0.15 \mathrm{D}$ of defocus at mid-high frequencies. The MTF's for other field angles have been computed as well but are not shown here. In summary, the agreement between experimental and simulated MTF curves is close for moderate field angles, being worse at the fovea but also at very large fields $\left(60^{\circ}\right)$ as a result of the mismatch between experimental and model astigmatism (see Fig. 4).

\section{DISCUSSION AND CONCLUSIONS}

A schematic eye model based on anatomical data, which had been designed previously to reproduce image quality on axis, ${ }^{14}$ has been transformed into a wide-angle model, simply by adding a spherical image surface that plays the role of the retina. This model was designed on the criterion of the minimum complexity necessary to reproduce, with a reasonable accuracy, the experimental average optical performance of the eye across the visual field. One of the most important features of this model is that all the geometrical parameters are anatomical, as we have strictly avoided any ad hoc fitting. In the original model, ${ }^{14}$ only some refractive indices (for near ultraviolet, blue, red, and near infrared) necessary to apply an interpolation formula for any visible wavelength had to be estimated, because of the lack of available data. (F urthermore, even the limited data available on dispersions are based on in vitro measurements, and hence they could differ from actual values in the living eye.) Nevertheless, these estimations were based on the criteria of (1) departing from experimental chromatic dispersion data and (2) fitting the experimental values of the LCA.

This simple schematic model does a good job of reproducing the wide-angle optical performance of an average eye. The experimental data available in the literature and results of computations with this eye model agree in that the optical quality is poor on axis for medium-large pupil sizes, remains relatively constant up to almost a $40^{\circ}$ field, and then deteriorates more rapidly at very large field angles. All these features are consistent with a wide-angle design. We want to remark that similar models ${ }^{15,18}$ are expected to show roughly similar monochromatic aberrations (for polychromatic light that they would need to incorporate appropriated refractive-index dispersions).

The agreement between aberrations of the eye model and average experimental data is quite reasonable in general. Astigmatism is possibly the aberration that displays the worst fit at large field angles $\left(>40^{\circ}\right)$. I I addition, this simple model does not predict on-axis coma or astigmatism, whereas foveal astigmatism is important in real eyes (and so is coma in certain subjects). Nevertheless, we believe that a few simple changes in the model, namely, including some pupil decentering and/or considering that the angle $\alpha$ between visual and optical axes is $\sim 5^{\circ}$, could help to obtain a better fit at the fovea. This will be a subject of future work. Furthermore, the model predictions for off-axis optical quality look more reliable than those for on-axis quality. A simple explanation for this could be that the effect of the lack of symmetry in real eyes may be crucial on axis (at the fovea) but comparatively much less important on the periphery.

As a first application of the model, we have performed numerical simulations of different experimental methods, designed to measure aberrations and selected from the available literature. This has permitted us a more direct comparison of our results with experimental data obtained with those methods. In many cases the model 
predicts significant biases between results of experimental measurements and actual ocular aberrations. This is the case when aberrations are measured in the object space (which has been the most common procedure) and then transformed into image-space aberrations through the Gaussian (paraxial) conjugate lens formula. Since experimental conditions are often far from being paraxial, especially off axis, this approach can yield substantially biased estimates, as the simulations have shown. We believe that this is an interesting result, with a potential influence on future experimental studies of ocular aberrations, as well as a quite direct and illustrative application of the model.

Another important and still open question is the role of the GRIN (or shell) structure of the lens in aberration control. It is clear that the GRIN lens in real eyes permits an increase in the refractive power of the crystalline lens, which otherwise would be too low on account of the small differences in refractive index between adjacent biological media that have a high water content. Thus the GRIN structure permits an increase in the effective refractive index of the lens and hence its power. ${ }^{1,2}$ However, the results of the present study suggest that the GRIN structure may play a secondary explicit role in optical quality (implicitly it plays an important first-order role, since the equival ent effective refractive index of the lens is the result of the GRIN structure). This permits a strong and convenient simplification of the model. On the contrary, asphericities, even with these simple conic models, do play a crucial role in keeping aberrations within reasonable limits. On axis, spherical surfaces would predict a much larger spherical aberration, ${ }^{14}$ and off axis Lotmar and Lotmar have found a similar result for astigmatism. ${ }^{16}$ Nevertheless, we expect to conduct an explicit study of the relative contribution of the GRIN structure and aspheric surfaces to the optical quality of the eye.

\section{ACKNOWLEDGMENT}

This research was supported by the Spanish Comisión Interministerial de Ciencia y Tecnología under grant TIC98-0925-C02-01.

Address correspondence to Rafael Navarro at the location on the title page or by phone, 34-91-590-1616; fax, 3491-564-5557; or e-mail, r.navarro@fresno.csic.es.

\section{REFERENCES AND NOTES}

1. A. Gullstrand, Appendix II in Handbuch der Physiologischen Optik, H. von Helmholtz, ed., 3rd ed. (Voss, Hamburg, 1909), Bd. 1, p. 299.

2. Y. Le Grand, La Dioptrique de I'Oeil et sa Correction, Tome I of Optique Physiologique (Masson, Paris, 1956); rev. ed. translated into English: Y. Le Grand and S. G. El Hage, Physiological Optics (Springer-Verlag, Berlin, 1980).

3. L. N. Thibos, M. Ye, X. Zhang, and A. Bradley, "The chromatic eye: a new reduced-eye model of ocular chromatic aberration in humans," Appl. Opt. 31, 3594-3600 (1992)

4. L. N. Thibos, M. Ye, X. Zhang, and A. Bradley, "Spherical aberration of the reduced schematic eye with elliptical refracting surface," Optom. Vision Sci. 74, 548-556 (1997).

5. Y. Wang and L. N. Thibos, "Oblique (off-axis) astigmatism of the reduced schematic eye with elliptical refracting surface," Optom. Vision Sci. 74, 557-562 (1997).

6. O. Pomerantzeff, M. Pankratov, G.-J. Wang, and P. Dufault, "Wide-angle optical model of the eye," Am. J . Optom. Physiol. Opt. 61, 166-176 (1984).

7. F. W. Fitzke, "A new schematic eye and its applications to psychophysics," presented at the Optical Society of America Topical Meeting on Recent Advances in Vision, Sarasota, Fla., April 30-May 3, 1980.

8. H.-L. Liou and N. A. Brennan, "Anatomically accurate, finite model eye for optical modeling," J . Opt. Soc. Am. A 14, 1684-1695 (1997).

9. D. A. Atchison and G. Smith, "Continuous gradient index and shell models of the human lens," Vision Res. 35, 25292538 (1995).

10. H. C. Howland and B. Howland, "A subjective method for the measurement of the monochromatic aberrations of the eye," J . Opt. Soc. Am. 67, 1508-1518 (1977).

11. J. Liang, B. Grimm, S. Goelz, and J. F. Bille, "Objective measurement of wave aberrations of the human eye with the use of a Hartmann-Shack wave-front sensor," J . Opt. Soc. Am. A 11, 1949-1957 (1994); see also J . Liang and D. R. Williams, "Aberrations and retinal image quality of the normal human eye," J. Opt. Soc. Am. A 14, 2873-2883 (1997).

12. R. Navarro and M. A. Losada, "Aberrations and relative efficiency of ray pencils in the living human eye," Optom. Vision Sci. 74, 540-547 (1997).

13. J. C. He, S. Marcos, R. H. Webb, and S. A. Burns, "Measurement of the wave-front aberration of the eye by a fast psychophysical procedure,"J . Opt. Soc. Am. A 15, 1-8 (1998).

14. R. Navarro, J. Santamaría, and J . Bescós, "Accommodationdependent model of the human eye with aspherics,"J . Opt. Soc. Am. A 2, 1273-1281 (1985).

15. W. Lotmar, "Theoretical eye model with aspherics,"J . Opt. Soc. Am. 61, 1522-1529 (1971).

16. W. Lotmar and T. Lotmar, "Peripheral astigmatism in the human eye: experimental data and theoretical model predictions," J . Opt. Soc. Am. 64, 510-513 (1974).

17. M. C. M. Dunne and D. A. Barnes, "Modelling oblique astigmatism in eyes with known peripheral refraction and optical dimensions," Ophthalmic Physiol. Opt. 10, 46-48 (1990).

18. A. C. Kooijman, "Light distribution on the retina of a wideangle theoretical eye," J. Opt. Soc. Am. 73, 1544-1550 (1983).

19. L. N. Thibos, A. Bradley, D. L. Still, X. Zhang, and P. A. Howarth, "Theory and measurement of ocular chromatic aberration," Vision Res. 30, 33-49 (1990).

20. T. Welford, Aberrations of Optical Systems (Hilger, London, 1986).

21. M. Koomen, R. Tousey, and R. Scolnik, "The spherical aberration of the eye," J . Opt. Soc. Am. 39, 370-376 (1949).

22. W. N. Charman and G. Walsh, "The optical phase transfer function of the eye and the perception of spatial phase," Vision Res. 25, 619-623 (1985).

23. A. van Meeteren, "Calculations on the optical modulation transfer function of the human eye," Opt. Acta 21, 395-412 (1974).

24. R. Navarro, E. Moreno, and C. Dorronsoro, "Monochromatic aberrations and point spread functions of the human eye across the visual field," J . Opt. Soc. Am. A 15, 2522-2529 (1998).

25. M. S. Smirnov, "Measurement of the wave aberration of the human eye," Biofizika 6, 687-703 (1961); [Biophysics (USSR) 6, 776-795 (1962]. That basic idea was later adopted by different authors: M. C. W. Campbell, E. H. Harrison, and P. Simonet, "Psychophysical measurement of the blur on the retina due to optical aberrations of the eye," Vision Res. 30, 1587-1602 (1990); R. H. Webb, C. M. Penney, and K. P. Thompson, "Measurement of ocular local wavefront distortion with a spatially resolved refractometer," Appl. Opt. 31, 3678-3686 (1992).

26. F. Rempt, J. Hoogerheide, and W. P. H. Hoogenboom, "Peripheral retinoscopy and the skiagram," Ophthalmologica 162, 1-10 (1971). 
27. M. C. Rynders, R. Navarro, and M. A. Losada, "Objective measurement of the off-axis longitudinal chromatic aberration in the human eye," Vision Res. 37, 513-521 (1997).

28. Y. U. Ogboso and H. E. Bedell, "Magnitude of lateral chromatic aberration across the retina of the human eye," J . Opt. Soc. Am. A 4, 1666-1672 (1987).
29. R. Navarro, P. Artal, and D. R. Williams, "Modulation transfer of the human eye as a function of retinal eccentricity," J . Opt. Soc. Am. A 10, 201-212 (1993).

30. D. R. Williams, P. Artal, R. Navarro, M. J. McMahon, and D. H. Brainard, "Off-axis optical quality and retinal sampling in the human eye," Vision Res. 36, 1103-1114 (1996). 